University of Texas at El Paso

ScholarWorks@UTEP

\title{
Estimating Correlation under Interval and Fuzzy Uncertainty: Case of Hierarchical Estimation
}

\author{
Ali Jalal-Kamali \\ The University of Texas at El Paso, ajalalkamali@miners.utep.edu
}

Follow this and additional works at: https://scholarworks.utep.edu/cs_techrep

Part of the Computer Sciences Commons, and the Mathematics Commons

Comments:

Technical Report: UTEP-CS-12-17

Published in Proceedings of the Annual Conference of the North American Fuzzy Information

Processing Society NAFIPS'2012, Berkeley, California, August 6-8, 2012.

\section{Recommended Citation}

Jalal-Kamali, Ali, "Estimating Correlation under Interval and Fuzzy Uncertainty: Case of Hierarchical Estimation" (2012). Departmental Technical Reports (CS). 695.

https://scholarworks.utep.edu/cs_techrep/695

This Article is brought to you for free and open access by the Computer Science at ScholarWorks@UTEP. It has been accepted for inclusion in Departmental Technical Reports (CS) by an authorized administrator of ScholarWorks@UTEP. For more information, please contact Iweber@utep.edu. 


\title{
Estimating Correlation under Interval and Fuzzy Uncertainty: Case of Hierarchical Estimation
}

\author{
Ali Jalal-Kamali \\ Department of Computer Science \\ University of Texas at El Paso \\ El Paso, TX 79968 \\ Email: ajalalkamali@miners.utep.edu
}

\begin{abstract}
In many situations, we are interested in finding the correlation $\rho$ between different quantities $x$ and $y$ based on the values $x_{i}$ and $y_{i}$ of these quantities measured in different situations $i$. The correlation is easy to compute when we know the exact sample values $x_{i}$ and $y_{i}$. In practice, the sample values come from measurements or from expert estimates; in both cases, the values are not exact. Sometimes, we know the probabilities of different values of measurement errors, but in many cases, we only know the upper bounds $\Delta_{x i}$ and $\Delta_{y i}$ on the corresponding measurement errors. In such situations, after we get the measurement results $\widetilde{x}_{i}$ and $\widetilde{y}_{i}$, the only information that we have about the actual (unknown) values $x_{i}$ and $y_{i}$ is that they belong to the corresponding intervals $\left[\widetilde{x}_{i}-\Delta_{x i}, \widetilde{x}_{i}+\Delta_{x i}\right]$ and $\left[\widetilde{y}_{i}-\Delta_{y i}, \widetilde{y}_{i}+\Delta_{y i}\right]$. For expert estimates, we get different intervals corresponding to different degrees of certainty - i.e., fuzzy sets. Different values of $x_{i}$ and $y_{i}$ lead, in general, to different values of the correlation $\rho$. It is therefore desirable to find the range $[\rho, \bar{\rho}]$ of possible values of the correlation when $x_{i}$ and $y_{i}$ take values from the corresponding intervals. In general, the problem of computing this range is NP-hard. In this paper, we provide a feasible (= polynomial-time) algorithm for computing at least one of the endpoints of this interval: for computing $\bar{\rho}$ when $\bar{\rho}>0$ and for computing $\underline{\rho}$ when $\underline{\rho}<0$.
\end{abstract}

\section{INTRODUCTION}

Need for correlation. In many practical situations, it is desirable to know which quantities are independent and which are correlated - positively or negatively.

To estimate the correlation between the quantities $x$ and $y$, we repeatedly measure the values $x_{i}$ and $y_{i}$ of both quantities in different situations $i$. The correlation $\rho$ is then estimated as the ratio $\rho=\frac{C}{\sqrt{V_{x}} \cdot \sqrt{V_{y}}}$, of the covariance $C$ to the product of standard deviations $\sqrt{V_{x}}$ and $\sqrt{V_{y}}$. Covariance and standard deviations, in their turn, are defined as follows:

$$
\begin{gathered}
C=\frac{1}{n} \cdot \sum_{i=1}^{n}\left(x_{i}-E_{x}\right) \cdot\left(y_{i}-E_{y}\right)=\frac{1}{n} \cdot \sum_{i=1}^{n} x_{i} \cdot y_{i}-E_{x} \cdot E_{y}, \\
V_{x}=\frac{1}{n} \cdot \sum_{i=1}^{n}\left(x_{i}-E_{x}\right)^{2}, \quad V_{y}=\frac{1}{n} \cdot \sum_{i=1}^{n}\left(y_{i}-E_{y}\right)^{2},
\end{gathered}
$$

and the means $E_{x}$ and $E_{y}$ are estimated as follows:

$$
E_{x}=\frac{1}{n} \cdot \sum_{i=1}^{n} x_{i}, \quad E_{y}=\frac{1}{n} \cdot \sum_{i=1}^{n} y_{i} .
$$

Need to take into account interval uncertainty. The values $x_{i}$ and $y_{i}$ used to estimate correlation come from measurements, and measurements are never absolutely accurate: the measurement results $\widetilde{x}_{i}$ and $\widetilde{y}_{i}$ are, in general, different from the actual (unknown) values $x_{i}$ and $y_{i}$ of the corresponding quantities. As a result, the value $\widetilde{\rho}$ estimated based on these measurement results is, in general, different from the ideal value $\rho$ which we would get if we could use the actual values $x_{i}$ and $y_{i}$. It is therefore desirable to determine how accurate is the resulting estimate.

Sometimes, we know the probabilities of different values of measurement errors $\Delta x_{i} \stackrel{\text { def }}{=} \widetilde{x}_{i}-x_{i}$ and $\Delta y_{i} \stackrel{\text { def }}{=} \widetilde{y}_{i}-y_{i}$. However, in many cases, we do not know these probabilities, we only know the upper bounds $\Delta_{x i}$ and $\Delta_{y i}$ on the corresponding measurement errors: $\left|\Delta x_{i}\right| \leq \Delta_{x i}$ and $\left|\Delta y_{i}\right| \leq \Delta_{y i}$; see, e.g., [5]. In this case, the only information that we have about the actual values $x_{i}$ and $y_{i}$ is that they belong to the corresponding intervals $\left[\underline{x}_{i}, \bar{x}_{i}\right]=\left[\widetilde{x}_{i}-\Delta_{x i}, \widetilde{x}_{i}+\Delta_{x i}\right]$ and $\left[\underline{y}_{i}, \bar{y}_{i}\right]=\left[\widetilde{y}_{i}-\Delta_{y i}, \widetilde{y}_{i}+\Delta_{y i}\right]$. Different values $x_{i} \in\left[\underline{x}_{i}, \bar{x}_{i}\right]$ and $y_{i} \in\left[\underline{y}_{i}, \bar{y}_{i}\right]$ lead, in general, to different values of the covariance. It is therefore desirable to find the range of all possible values of the covariance $\rho$ :

$$
\begin{gathered}
{[\underline{\rho}, \bar{\rho}]=} \\
\left\{\rho\left(x_{1}, \ldots, x_{n}, y_{1}, \ldots, y_{n}\right): x_{i} \in\left[\underline{x}_{i}, \bar{x}_{i}\right], y_{i} \in\left[\underline{y}_{i}, \bar{y}_{i}\right]\right\} .
\end{gathered}
$$

Case of expert uncertainty, and how the corresponding computations can be reduced to the interval case. An expert usually describes his/her uncertainty by using words from the natural language, like "most probably, the value of the quantity is between 6 and 7, but it is somewhat possible to have values between 5 and 8 ". To formalize this knowledge, it is natural to use fuzzy set theory, in which, for every value $x_{i}$, we have a fuzzy set $\mu_{i}\left(x_{i}\right)$ which describes the expert's knowledge about $x_{i}$. An alternative user-friendly way to represent a fuzzy set is by using its $\alpha$-cuts $\mathbf{x}_{i}(\alpha)=\left\{x_{i} \mid \mu_{i}\left(x_{i}\right) \geq \alpha\right\}$. It is known (see, e.g., [4]) that for any function $y=f\left(x_{1}, \ldots, x_{n}\right)$, the $\alpha$-cut of $y$ is equal to

$$
\mathbf{y}(\alpha)=\left\{f\left(x_{1}, \ldots, x_{n}\right): x_{1} \in \mathbf{x}_{1}(\alpha), \ldots, x_{n} \in \mathbf{x}_{n}(\alpha)\right\} .
$$

Thus, from the computational viewpoint, the problem of estimating $\rho$ under fuzzy uncertainty can be reduced to several 
similar problems for interval uncertainty - interval problems corresponding to different values $\alpha$. In view of this reduction, in the following text, we will concentrate on estimating the correlation under interval uncertainty.

What is known. The problem of estimating correlation under interval uncertainty is, in general, NP-hard [3]. This means, crudely speaking, that unless $\mathrm{P}=\mathrm{NP}$ (which most computable scientists believe to be impossible), no feasible (i.e., no polynomial-time) algorithm is possible that would always compute the range of the corresponding characteristic under interval uncertainty.

In [1], we showed that while we cannot have an efficient algorithm for computing both bounds $\rho$ and $\bar{\rho}$, we can effectively compute (at least) one of the bounds. We consider the case that we have weights $w_{i}$ for corresponding values $x_{i}$ and $y_{i}$ and we show that our claim still holds. Specifically, that we can compute $\bar{\rho}$ when $\bar{\rho}>0$ and we can compute $\rho$ when $\rho<0$. This means that, in the case of a non-degenerate interval $[\rho, \bar{\rho}]$ (i.e., $\rho<\bar{\rho}$ ):

- when $\bar{\rho} \leq 0$, we compute the lower endpoint $\rho$;

- when $0 \leq \rho$, we compute the upper endpoint $\overline{\bar{\rho}}$;

- in all remaining cases, when $\rho<0<\bar{\rho}$, we compute both lower endpoint $\underline{\rho}$ and $\bar{\rho}$.

Need to take into account that the estimation is usually hierarchical. In some practical situations, e.g., when processing the census results, we do not process all the census data, what we usually do is we first combine the data by town, then combine town data into state-wide date, etc.; see, e.g., [2], [6].

In general, on each stage, the data points are divided into groups $I_{1}, \ldots, I_{m}$, and instead of directly processing all the data points, we process the results of previous processing within each of these $m$ groups. For example, in the previous processing, we have compute the averages $E_{x j}=\frac{1}{n_{j}} \cdot \sum_{i \in I_{j}} x_{i}$ over each group. Now, the overall average $E_{x}$ can be described as

$$
E_{x}=\frac{1}{n} \cdot \sum_{i=1}^{n} x_{i}=\frac{1}{n} \cdot \sum_{j=1}^{m} \sum_{i \in I_{j}} x_{i}=\sum_{j=1}^{m} p_{j} \cdot E_{x j}
$$

i.e.,

$$
E_{x}=\sum_{j=1}^{m} p_{j} \cdot E_{x j}
$$

where we denoted $p_{j} \stackrel{\text { def }}{=} \frac{n_{j}}{n}$. Similarly [6],

$$
\begin{gathered}
E_{y}=\sum_{j=1}^{m} p_{j} \cdot E_{y j}, \\
V_{x}=\sum_{j=1}^{m} p_{j} \cdot\left(E_{x j}-E_{x}\right)^{2}+\sum_{j=1}^{m} p_{j} \cdot V_{x j}, \\
V_{y}=\sum_{j=1}^{m} p_{j} \cdot\left(E_{y j}-E_{y}\right)^{2}+\sum_{j=1}^{m} p_{j} \cdot V_{y j},
\end{gathered}
$$

where $V_{x j}$ and $V_{y j}$ are sample variances within the corresponding group. For covariance, we have

$$
C=\sum_{j=1}^{m} p_{j} \cdot\left(E_{x j}-E_{x}\right) \cdot\left(E_{y j}-E_{y}\right)+\sum_{j=1}^{m} p_{j} \cdot C_{j},
$$

where $C_{j}$ is the covariance over each group. Finally, we compute correlation $\rho$ as

$$
\rho=\frac{C}{\sqrt{V_{x}} \cdot \sqrt{V_{y}}}
$$

Hierarchical estimation under interval uncertainty. In the ideal case, for each group $j$, we know the values $p_{j}, E_{x j}, E_{y j}$, $V_{x j}, V_{y j}$, and $C_{j}$. Based on these values, we compute $E, V_{x}$, $V_{y}, C$, and finally, the correlation $\rho$. In practice, we often only know the values $x_{i}$ and $y_{i}$ with interval uncertainty. As a result, for each group $j$, instead of the exact value of the each of the above characteristics, we only know the interval of its possible values, i.e., we know the intervals $\mathbf{E}_{x j}, \mathbf{E}_{x j}, \mathbf{E}_{y j}, \mathbf{V}_{x j}, \mathbf{V}_{y j}$, and $\mathbf{C}_{j}$. Different values from these intervals lead, in general, to different correlation values $\rho$. It is therefore desirable to find the range $[\rho, \bar{\rho}]$ of possible values of the correlation obtained by formulas (1)-(6).

What we do in this paper. In this paper, we show that for such a hierarchical estimation, it is still possible to feasibly compute at least one of the endpoints of the interval of possible values of the correlation $\rho$.

\section{Main Result And The CorResponding Algorithm}

Main Result. There exists a polynomial-time algorithm that, given $m$ numbers $p_{j}$ and $m$ groups of intervals $\mathbf{E}_{x j}, \mathbf{E}_{x j}, \mathbf{E}_{y j}$, $\mathbf{V}_{x j}, \mathbf{V}_{y j}$, and $\mathbf{C}_{j}$, computes (at least) one of the endpoint of the interval $[\rho, \bar{\rho}]$ of possible values of the correlation $\rho$ :

- it computes $\bar{\rho}$ if $\bar{\rho}>0$, and

- it computes $\underline{\rho}$ if $\underline{\rho}<0$.

Reducing minimum to maximum. One can easily see that when we change the signs of $E_{y j}$ and $C_{j}$, the correlation changes sign as well. It is known that

$$
\min f(x)=-\max (-f(x)) .
$$

So, if if we know how to compute the largest value $\bar{\rho}$ when this value is positive, we can then compute the smallest value $\underline{\rho}$ when this value is negative, by taking intervals $\mathbf{E}_{y j}^{\prime}=-\mathbf{E}_{y j}$ and $\mathbf{C}_{j}^{\prime}=-\mathbf{C}_{j}$, computing the corresponding bound $\overline{\rho^{\prime}}$, and then taking $\rho=-\overline{\rho^{\prime}}$.

Because of this reduction, in the following text, we will concentrate on computing the largest value $\bar{\rho}$.

Preliminary observation. In the ratio $\rho$, the dependence on $C_{j}$ is only in the numerator, and the dependence on $V_{x j}$ and $V_{y j}$ is only in the denominator. Thus, the ratio $\rho$ is the largest when each term $C_{j}$ attains its largest possible value $\bar{C}_{j}$, and when each term $V_{x j}$ and $V_{y j}$ attains its smallest possible value $\underline{V}_{x j}$ and $\underline{V}_{y j}$. So, in the following text, we will take $C_{j}=\bar{C}_{j}$, 
$V_{x j}=\underline{V}_{x j}$, and $V_{y j}=\underline{V}_{y j}$, and consider only the dependence on $X_{x j}$ and $E_{y j}$.

Algorithm. For each $j$ from 1 to $m$, the corresponding box $\left[\underline{E}_{x j}, \bar{E}_{x j}\right] \times\left[\underline{y}_{i}, \bar{E}_{y j}\right]$ has four vertices: $\left(\underline{E}_{x j}, \underline{E}_{y j}\right)$, $\left(\underline{E}_{x j}, \bar{E}_{y j}\right),\left(\bar{E}_{x j}, \underline{E}_{y j}\right)$, and $\left(\bar{E}_{x j}, \bar{E}_{y j}\right)$. So, totally, we have $4 n$ vertices.

Let us consider all 4-tuples consisting of two vertices and two signs. For each pair of vertices, there are nine possible combinations of two,+- , or 0 signs: $(-,-),(-, 0),(-,+)$, $(0,-),(0,0),(0,+),(+,-),(+, 0)$, and $(+,+)$.

For each 4-tuple, if the first sign is not 0 , we move the first vertex slightly along the $x$ axis in the direction determined by the first sign, i.e.:

- slightly increase $x$ if the sign is + and

- slightly decrease $x$ if the sign is -.

Here, "slightly" means that the change is much smaller than the smallest difference between distinct values $E_{x j}$ and $E_{y j}$.

Then, if the second sign is not 0 , we move the second vertex slightly along the $x$ axis in the direction determined by the second sign. Thus, we get two points on the $(x, y)$ plane. We can then form a straight line going through these two points.

Now, we select two 4-tuples, and form two lines. We will call the first line representative $x$-line, and the second line representative $y$-line.

If we selected the same line as the representative $x$-line and the representative $y$-line, then we check whether this line intersects each of $n$ boxes. If it does, then $\bar{\rho}=1$. If this line does not have a common point with one of the boxes, we dismiss this selection, and continue with other selections.

Let us explain the algorithm in the cases when the representative $x$-line and the representative $y$-line are different. The representative $x$-line divides the plane into two semi-planes:

- the points above this line, i.e., the points $(x, y)$ for which the $y$ coordinate is larger than the $y$-value of the point on the $x$-line with the same $x$ coordinate, and

- the points below this line, i.e., the points $(x, y)$ for which the $y$ coordinate is smaller than the $y$-value of the point on the $x$-line with the same $x$ coordinate.

The representative $y$-line similarly divides the plane into two semi-planes:

- the points to the right of this line, i.e., the points $(x, y)$ for which the $x$ coordinate is larger than the $x$-value of the point on the $x$-line with the same $y$ coordinate, and

- the points to the left of this line, i.e., the points $(x, y)$ for which the $x$ coordinate is smaller than the $x$-value of the point on the $y$-line with the same $y$ coordinate.

Based on where each of the vertices is with respect to these two lines, we can tell the relation of each box $\left[\underline{E}_{x j}, \bar{E}_{x j}\right] \times$ $\left[\underline{E}_{y j}, \bar{E}_{y j}\right]$ with respect to each line.

The lines that we computed are "representatives" of the actual lines that we will be using, in the sense that the actual lines will have the exact same relation to each of the $n$ boxes. Let us describe the corresponding actual lines as follows:

- the actual $x$-line has the form $y=E_{y}+k_{x} \cdot\left(x-E_{x}\right)$, and
- the actual $y$-line has the form $x=E_{x}+k_{y} \cdot\left(y-E_{y}\right)$, where $E_{x}, E_{y}, k_{x}$, and $k_{y}$ are to-be-determined real numbers.

For each box $\left[\underline{E}_{x j}, \bar{E}_{x j}\right] \times\left[\underline{E}_{y j}, \bar{E}_{y j}\right]$, based on its location in comparison to the representative lines, we select the values $E_{x j}$ and $E_{y j}$ as follows:

- If the whole box is above the representative $x$-line, we take $E_{x j}=\bar{E}_{x j}$. On the resulting segment $\left\{\bar{E}_{x j}\right\} \times$ $\left[\underline{E}_{y j}, \bar{E}_{y j}\right]$, we select the point which is the closest to the actual $y$-line:

- if the whole segment is to the right of the representative $y$-line, we select $E_{y j}=\underline{E}_{y j}$;

- if the whole segment is to left of the representative $y$-line, we select $E_{y j}=\bar{E}_{y j}$;

- if the segment intersects with the representative $y$ line, we select the value $E_{y j}$ corresponding to the intersection point between the segment and the actual $y$-line.

- If the whole box is below the representative $x$-line, we take $E_{x j}=\underline{E}_{x j}$. On the resulting segment $\left\{\underline{E}_{x j}\right\} \times$ $\left[\underline{E}_{y j}, \bar{E}_{y j}\right]$, we select the point which is the closest to the actual $y$-line:

- if the whole segment is to the right of the representative $y$-line, we select $E_{y j}=\underline{E}_{y j}$;

- if the whole segment is to left of the representative $y$-line, we select $E_{y j}=\bar{E}_{y j}$;

- if the segment intersects with the representative $y$ line, we select the value $E_{y j}$ corresponding to the intersection point between the segment and the actual $y$-line.

- If the whole box is to the right of the representative $y$-line, we take $E_{y j}=\underline{E}_{y j}$. On the resulting segment $\left[\underline{E}_{x j}, \bar{E}_{x j}\right] \times\left\{\underline{E}_{y j}\right\}$, we select the point which is the closest to the actual $x$-line:

- if the whole segment is above the representative $x$ line, we select $E_{x j}=\underline{E}_{x j}$;

- if the whole segment is below the representative $x$ line, we select $E_{x j}=\bar{E}_{x j}$;

- if the segment intersects with the representative $x$ line, we select the value $E_{x j}$ corresponding to the intersection point between this segment and the actual $x$-line.

- If the whole box is to the left of the representative $y$ line, we take $E_{y j}=\bar{E}_{y j}$. On the resulting segment $\left[\underline{E}_{x j}, \bar{E}_{x j}\right] \times\left\{\bar{E}_{y j}\right\}$, we select the point which is the closest to the actual $x$-line:

- if the whole segment is above the representative $x$ line, we select $E_{x j}=\underline{E}_{x j}$;

- if the whole segment is below the representative $x$ line, we select $E_{x j}=\bar{E}_{x j}$;

- if the segment intersects with the representative $x$ line, we select the value $E_{x j}$ corresponding to the intersection point between the segment and the actual $x$-line.

- The only remaining case is when the box contains the intersection point $\left(E_{x}, E_{y}\right)$ of the actual $x$ - and $y$-lines. 
Thus, for each $j$ and for each of the values $E_{x j}$ and $E_{y j}$, we get an explicit expression in terms of the four parameters $E_{x}$, $E_{y}, k_{x}$ and $k_{y}$ (the parameters that describe the actual $x$ - and $y$-lines).

By substituting these expressions for $E_{x j}$ and $E_{y j}$ into the following formulas, we get a system of four equations with four unknowns $E_{x}, E_{y}, k_{x}$ and $k_{y}$ :

$$
\begin{gathered}
E_{x}=\sum_{j=1}^{m} p_{j} \cdot E_{x j} ; \\
E_{y}=\sum_{j=1}^{m} p_{j} \cdot E_{y j} ; \\
\sum_{i=1}^{n} p_{j} \cdot E_{x j} \cdot E_{y j}-E_{x} \cdot E_{y}+\sum_{j=1}^{m} p_{j} \cdot \bar{C}_{j}= \\
k_{x} \cdot\left(\sum_{j=1}^{m} p_{j} \cdot\left(E_{x j}-E_{x}\right)^{2}+\sum_{j=1}^{m} p_{j} \cdot \underline{V}_{x j}\right) \\
\sum_{j=1}^{n} p_{j} \cdot E_{x j} \cdot E_{y j}-E_{x} \cdot E_{y}+\sum_{j=1}^{m} p_{j} \cdot \bar{C}_{j}= \\
k_{y} \cdot\left(\sum_{j=1}^{m} p_{j} \cdot\left(E_{y j}-E_{y}\right)^{2}+\sum_{j=1}^{m} p_{j} \cdot \underline{V}_{y j}\right) .
\end{gathered}
$$

Once we solve this system, we get one or several possible solutions. For each of these solutions, we can form the corresponding actual $x$ - and $y$-lines.

Then, we check whether each of $4 n$ vertices is in the same relation to the resulting two lines and to the representative $x$ - and $y$-lines, i.e., e.g., that each vertex is above, below, or on the actual $x$-line if and only if it is, correspondingly, above, below, or on the corresponding representative $x$-line, and that the same property holds for the $y$-lines. If at least one vertex is in a different relation, we dismiss this solution. Otherwise, we compute the value of the correlation $\rho$ based on the corresponding values $E_{x j}$ and $E_{y j}$.

The largest of all the values $\rho$ corresponding to all possible pairs of tuples is then returned as the desired value $\bar{\rho}$.

Comment. For each pair of lines, for each $j$, according to our algorithm, as the appropriate value of $E_{x j}$, we make one of the following four selections:

- sometimes, we select a known value $\underline{E}_{x j}$;

- sometimes, we select a know value $\bar{E}_{x j}$;

- sometimes, we select the value $E_{x j}=E_{x}$ (which is not a priori known, it is one of the four variables that we need to determine), and

- sometimes, we select a value $E_{x j}$ that lies on the $x$-line $y=E_{y}+k_{x} \cdot\left(E_{x j}-E_{x}\right)$, i.e., a value

$$
E_{x j}=E_{x}+K_{x} \cdot\left(E_{y j}-E_{y}\right),
$$

where $K_{x} \stackrel{\text { def }}{=} \frac{1}{k_{x}}=\frac{V_{x}}{C}$.
In general, each expression $E_{x j}$ is a linear combination of a constant and the unknowns $E_{x}, K_{x}$, and $K_{x} \cdot E_{y}$. According to the algorithm, for each $i$, it takes a finite number of computational steps to check the corresponding conditions and, based on the results of this checking, to find the appropriate value $E_{x j}$. Similarly, each expression $E_{y j}$ is a linear combination of a constant and the unknowns $E_{y}, K_{y}$, and $K_{y} \cdot E_{x}$.

Substituting these expressions for $E_{x j}$ and $E_{y j}$ into the four equations for the unknowns $E_{x}, E_{y}, K_{x}$, and $K_{y}$, we conclude that:

- the equation (7) for $E_{x}$ is transformed into equating a linear combination of $E_{x}, K_{x}$, and $K_{x} \cdot E_{y}$ to zero;

- the equation (8) for $E_{y}$ is transformed into equating a linear combination of $E_{y}, K_{y}$, and $K_{y} \cdot E_{x}$, to zero;

- the equations (9) and (10) (corresponding to $k_{x} \cdot V_{x}=$ $k_{y} \cdot V_{y}=C$ ) are transformed into equating a linear combination of terms of order $\leq 4$ in terms of the unknowns.

As a result, to find the four unknown $E_{x}, E_{y}, K_{x}$, and $K_{y}$, we get a system of four polynomial equations of order $\leq 4$. The amount of computation time which is needed to solve this system does not depend on the size $m$ of the input, so in terms of dependence on this size, we need $O(1)$ time.

\section{Proof of the Main Result}

Proof that the above algorithm is polynomial time. Before we prove that the algorithm is correct, let us first prove that it is indeed a polynomial time algorithm.

We have $4 m$ possible vertices, so we have $O\left(\mathrm{~m}^{2}\right)$ possible pairs of vertices - and thus, $O\left(\mathrm{~m}^{2}\right)$ possible 4-tuples. Thus, we have $O\left(\mathrm{~m}^{2}\right)$ possible representative $x$-lines, and we also have $O\left(\mathrm{~m}^{2}\right)$ representative $y$-lines. In our algorithms, we consider pairs consisting of a representative $x$-line and a representative $y$-line. Since we have $O\left(\mathrm{~m}^{2}\right) x$-lines and we have $O\left(\mathrm{~m}^{2}\right) y$ lines, we therefore have $O\left(\mathrm{~m}^{2}\right) \cdot O\left(\mathrm{~m}^{2}\right)=O\left(\mathrm{~m}^{4}\right)$ possible pairs consisting of a representative $x$-line and a representative $y$-line.

For each pair of lines, we perform the following computations:

- First, need a constant number of steps to find the expression for each of $m$ values $E_{x j}$ and each of $n$ values $E_{y j}$ in terms of the parameters $E_{x}, E_{y}, K_{x}$, and $K_{y}$. So, we need $O(m)$ steps to find these expressions for all $j$.

- Then, we need linear time $O(m)$ to form the corresponding systems of four equations with four unknowns and constant time $O(1)$ to solve this system.

- Once this system is solved, and we know the corresponding values $E_{x}, E_{y}, k_{x}$, and $k_{y}$, we need:

- a linear time $O(m)$ to check whether each of $4 m=$ $O(m)$ vertices is in the right position with respect to the corresponding lines, and,

- if needed, linear time $O(m)$ to compute the corresponding value of the correlation $\rho-$ by using the above explicit formulas (1)-(6) describing how the correlation $\rho$ depends on $E_{x j}$ and $E_{y j}$. 
Totally, for each pair of lines, we need

$$
O(m)+O(m)+O(1)+O(m)+O(m)=O(m)
$$

computational steps.

We need $O(m)$ steps for each of $O\left(\mathrm{~m}^{4}\right)$ pairs of lines. Thus, the total computation time of this algorithm is $O\left(\mathrm{~m}^{4}\right) \cdot O(\mathrm{~m})=$ $O\left(m^{5}\right)$ - which is indeed polynomial in the size $m$ of the problem.

Case when the representative $x$-line coincides with the representative $y$-line. If this common line intersects with all $m$ boxes $\left[\underline{E}_{x j}, \bar{E}_{x j}\right] \times\left[\underline{E}_{y j}, \bar{E}_{y j}\right]$, then, for each box, we can select values $E_{x j}$ and $E_{y j}$ for which the corresponding point $\left(E_{x j}, E_{y j}\right)$ belongs to this line. Then, all selected values $\left(E_{x j}, E_{y j}\right)$ follow the same linear dependence $E_{y j}=E_{y}+k_{x} \cdot\left(E_{x j}-E_{x}\right)$ (as described by the common lines). Therefore, for this selection, the correlation is 1 . Since $\rho \leq 1$, this means that in this case, $\bar{\rho}=1$.

Remaining cases. Let us now prove that our algorithm is correct for all other cases, when the $x$ - and the $y$-lines are different.

When a function attains maximum on the interval: known facts from calculus. A function $f(x)$ defined on an interval $[\underline{x}, \bar{x}]$ attains its maximum either at one of its endpoints, or in some internal point of the interval. If it attains is maximum at a point $x \in(a, b)$, then its derivative at this point is 0 : $\frac{d f}{d x}=0$.

If it attains its maximum at the point $x=\underline{x}$, then we cannot have $\frac{d f}{d x}>0$, because then, for some point $x+\Delta x \in[\underline{x}, \bar{x}]$, we would have a larger value of $f(x)$. Thus, in this case, we must have $\frac{d f}{d x} \leq 0$.

Similarly, if a function $f(x)$ attains its maximum at the point $x=\bar{x}$, then we must have $\frac{d f}{d x} \geq 0$.

Computing the corresponding derivatives. Based on the expression we had for $E_{x}$, we conclude that $\frac{\partial E_{x}}{\partial E_{x j}}=p_{j}$ and similarly $\frac{\partial E_{y}}{\partial E_{y j}}=p_{j}$. Since the variance $V_{x}$ can be described in an equivalent form

$$
V_{x}=\sum_{j=1}^{m} p_{j} X_{x j}^{2}-E_{x}^{2}+\sum_{j=1}^{m} p_{j} \cdot \underline{V}_{x j},
$$

we get $\frac{\partial V_{x}}{\partial E_{x j}}=2 p_{j} \cdot\left(E_{x j}-E_{x}\right)$. Similarly, we get $\frac{\partial V_{y}}{\partial E_{y j}}=$ $2 \cdot p_{j} \cdot\left(E_{y j}-E_{y}\right)$. The covariance can be equivalently rewritten as

$$
C=\sum_{j=1}^{m} p_{j} \cdot E_{x j} \cdot E_{y j}-E_{x} \cdot E_{y}+\sum_{j=1}^{m} p_{j} \cdot \bar{C}_{j}
$$

hence

$$
\frac{\partial C}{\partial E_{x j}}=p_{j} \cdot\left(E_{y j}-E_{y}\right) \text { and } \frac{\partial C}{\partial E_{y j}}=p_{j} \cdot\left(E_{x j}-E_{x}\right) .
$$

So, for $\rho=\frac{C}{\sqrt{V_{x}} \cdot \sqrt{V_{y}}}$, we get

$$
\frac{\partial \rho}{\partial E_{x j}}=\frac{p_{j}}{\sqrt{V_{y}} \cdot V_{x}} \cdot\left[\left(E_{y j}-E_{y}\right) \cdot \sqrt{V_{x}}-C \cdot \frac{E_{x j}-E_{x}}{\sqrt{V_{x}}}\right] \text {. }
$$

Since the standard deviations are always non-negative, the sign of this derivative coincides with the sign of the value

$$
\left(E_{y j}-E_{y}\right) \cdot \sqrt{V_{x}}-C \cdot \frac{E_{x j}-E_{x}}{\sqrt{V_{x}}} .
$$

Dividing this expression by a positive value $\sqrt{V_{x}}$, we conclude that the sign of the derivative $\frac{\partial \rho}{\partial E_{x j}}$ coincides with the sign of the expression $\left(E_{y j}-E_{y}\right)-k_{x} \cdot\left(E_{x j}-E_{x}\right)$, where we denoted $k_{x} \stackrel{\text { def }}{=} \frac{C}{V_{x}}$.

Similarly, the sign of the derivative $\frac{\partial \rho}{\partial E_{y j}}$ coincides with the sign of the expression $\left(E_{x j}-E_{x}\right)-k_{y} \cdot\left(E_{y j}-E_{y}\right)$, where we denoted $k_{y} \stackrel{\text { def }}{=} \frac{C}{V_{y}}$.

Let us apply the known facts from calculus to this situation. Let $E_{x j}$ and $E_{y j}$ be the values from the corresponding boxes for which the correlation $\rho$ attains its largest possible value $\bar{\rho}>0$. Then, according to the above facts from calculus, we have one of the three possible situations:

$$
\begin{gathered}
\text { - } E_{x j} \in\left(\underline{E}_{x j}, \bar{E}_{x j}\right) \text { and } \frac{\partial \rho}{\partial E_{x j}}=0 \text {, i.e., } \\
E_{y j}=E_{y}+k_{x} \cdot\left(E_{x j}-E_{x}\right) ; \\
\text { - } E_{x j}=\underline{E}_{x j} \text { and } \frac{\partial \rho}{\partial E_{x j}} \leq 0 \text {, i.e., } \\
E_{y j} \leq E_{y}+k_{x} \cdot\left(E_{x j}-E_{x}\right) ; \\
\text { - } E_{x j}=\bar{E}_{x j} \text { and } \frac{\partial \rho}{\partial E_{x j}} \geq 0 \text {, i.e., } \\
E_{y j} \geq E_{y}+k_{x} \cdot\left(E_{x j}-E_{x}\right) .
\end{gathered}
$$

Here, $k_{x}$ has the same sign as the correlation, so $k_{x}>0$. Let us now consider possible locations of the box $\left[\underline{E}_{x j}, \bar{E}_{x j}\right] \times$ $\left[\underline{E}_{y j}, \bar{E}_{y j}\right]$ with respect to the $x$-line

$$
E_{y j}=E_{y}+k_{x} \cdot\left(E_{x j}-E_{x}\right) .
$$

$1^{\circ}$. The first case is when the whole box $\left[\underline{E}_{x j}, \bar{E}_{x j}\right] \times$ $\left[\underline{E}_{y j}, \bar{E}_{y j}\right]$ is above the $x$-line $E_{y j}=E_{y}+k_{x} \cdot\left(E_{x j}-E_{x}\right)$, i.e., when $E_{y j}>E_{y}+k_{x} \cdot\left(E_{x j}-E_{x}\right)$ for all $E_{y j} \in\left[\underline{E}_{y j}, \bar{E}_{y j}\right]$ and $E_{x j} \in\left[\underline{E}_{x j}, \bar{E}_{x j}\right]$. In this case, we cannot have $E_{x j} \in$ $\left(\underline{E}_{x j}, \bar{E}_{x j}\right)$ and $E_{x j}=\underline{E}_{x j}$, so we must have $E_{x j}=\bar{E}_{x j}$.

On the segment $E_{x j}=\bar{E}_{x j}$, we can apply the same argument about the dependence on $E_{y j}$ and conclude that we can have one of the three possible situations:

$$
\begin{array}{r}
-E_{y j} \in\left(\underline{E}_{y j}, \bar{E}_{y j}\right) \text { and } \frac{\partial \rho}{\partial E_{y j}}=0, \text { i.e., } \\
E_{x j}=E_{x}+k_{y} \cdot\left(E_{y j}-E_{y}\right) ;
\end{array}
$$




$$
\begin{gathered}
\text { - } E_{y j}=\underline{E}_{y j} \text { and } \frac{\partial \rho}{\partial E_{y j}} \leq 0 \text {, i.e., } \\
E_{x j} \leq E_{x}+k_{y} \cdot\left(E_{y j}-E_{y}\right) ; \\
\text { - } E_{y j}=\bar{E}_{y j} \text { and } \frac{\partial \rho}{\partial E_{y j}} \geq 0 \text {, i.e., } \\
E_{x j} \geq E_{x}+k_{y} \cdot\left(E_{y j}-E_{y}\right) .
\end{gathered}
$$

Here, $k_{y}$ has the same sign as the correlation, so $k_{y}>0$. Let us now consider possible locations of the segment $\left\{\bar{E}_{x j}\right\} \times$ $\left[\underline{E}_{y j}, \bar{E}_{y j}\right]$ in relation to the $y$-line $E_{x j}=E_{x}+k_{y} \cdot\left(E_{y j}-E_{y}\right)$.

$1.1^{\circ}$. The first subcase is when the whole segment is to the left of the $y$-line, i.e., when $E_{x j}<E_{x}+k_{y} \cdot\left(E_{y j}-E_{y}\right)$ for all $E_{y j} \in\left[\underline{E}_{y j}, \bar{E}_{y j}\right]$. In this case, we cannot have $E_{y j} \in$ $\left(\underline{E}_{y j}, \bar{E}_{y j}\right)$ and we cannot have $E_{y j}=\bar{E}_{y j}$, so we must have $E_{y j}=\underline{E}_{y j}$.

$1.2^{\circ}$. The second subcase is when the whole segment is to the right of the $y$-line, i.e., when $E_{x j}>E_{x}+k_{y} \cdot\left(E_{y j}-E_{y}\right)$ for all $E_{y j} \in\left[\underline{E}_{y j}, \bar{E}_{y j}\right]$. In this case, we cannot have $E_{y j} \in$ $\left(\underline{E}_{y j}, \bar{E}_{y j}\right)$ and we cannot have $E_{y j}=\underline{E}_{y j}$, so we must have $E_{y j}=\bar{E}_{y j}$.

$1.3^{\circ}$. The third subcase is when the segment intersects the $y$ line, i.e., when $E_{x j}=E_{x}+k_{y} \cdot\left(E_{y j}^{\prime}-E_{y}\right)$ for some $E_{y j}^{\prime} \in$ $\left[\underline{E}_{y j}, \bar{E}_{y j}\right]$. As we have mentioned, there are three possibility for the value $E_{y j}$ at which the correlation attains its maximum: the value for which $E_{x j}=E_{x}+k_{y} \cdot\left(E_{y j}-E_{y}\right)$, the value $\underline{E}_{y j}$, and the value $\bar{E}_{y j}$.

$1.3 .1^{\circ}$. In the first case (when $E_{x j}=E_{x}+k_{y} \cdot\left(E_{y j}-E_{y}\right)$ ), since $k_{y}>0$, there is only one value $E_{y j}=E_{y j}^{\prime}$.

$1.3 .2^{\circ}$. If $\underline{E}_{y j} \neq E_{y j}^{\prime}$, then $\underline{E}_{y j}<E_{y j}^{\prime}$, and thus,

$$
E_{x}+k_{y} \cdot\left(\underline{E}_{y j}-E_{y}\right)<E_{x}+k_{y} \cdot\left(E_{y j}^{\prime}-E_{y}\right)=E_{x j} .
$$

Thus, we have $E_{x j}>E_{x}+k_{y} \cdot\left(\underline{E}_{y j}-E_{y}\right)$, so we cannot have $E_{x j} \leq E_{x}+k_{y} \cdot\left(\underline{E}_{y j}-E_{y}\right)$, and therefore, the maximum cannot be attained for $E_{y j}=\underline{E}_{y j}$.

$1.3 .3^{\circ}$. If $\bar{E}_{y j} \neq E_{y j}^{\prime}$, then $E_{y j}^{\prime}<\bar{E}_{y j}$, and thus,

$E_{x j}=E_{x}+k_{y} \cdot\left(E_{y j}^{\prime}-E_{y}\right)<E_{x}+k_{y} \cdot\left(\bar{E}_{y j}-E_{y}\right)=E_{x j}$.

Thus, we have $E_{x j}<E_{x}+k_{y} \cdot\left(\bar{E}_{y j}-E_{y}\right)$, so we cannot have $E_{x j} \leq E_{x}+k_{y} \cdot\left(\bar{E}_{y j}-E_{y}\right)$, and therefore, maximum cannot be attained for $E_{y j}=\bar{E}_{y j}$.

1.3. $4^{\circ}$. Therefore, in this third subcase, maximum can only be attained at the point on the $y$-line.

$2^{\circ}$. The second case is when the whole box $\left[\underline{E}_{x j}, \bar{E}_{x j}\right] \times$ $\left[\underline{E}_{y j}, \bar{E}_{y j}\right]$ is below the $x$-line $E_{y j}=E_{y}+k_{x} \cdot\left(E_{x j}-E_{x}\right)$, i.e., when $E_{y j}<E_{y}+k_{x} \cdot\left(E_{x j}-E_{x}\right)$ for all $E_{y j} \in\left[\underline{E}_{y j}, \bar{E}_{y j}\right]$ and $E_{x j} \in\left[\underline{E}_{x j}, \bar{E}_{x j}\right]$. In this case, we cannot have $E_{x j} \in$ $\left(\underline{E}_{x j}, \bar{E}_{x j}\right)$ and we cannot have $E_{x j}=\bar{E}_{x j}$, so we must have $E_{x j}=\underline{E}_{x j}$.
On the segment $E_{x j}=\underline{E}_{x j}$, we can apply the same argument about the dependence on $E_{y j}$ as in Part 1 of this proof and come with the same conclusions.

$3^{\circ}$. Same arguments apply if the whole box is fully to the left or to the right of the $y$-line. In this case, we have $E_{y j}=\bar{E}_{y j}$ or $E_{y j}=\underline{E}_{y j}$.

$4^{\circ}$. The only remaining case is when the box intersects both with the $x$-line and with the $y$-line. In this case, similar to Part 1.3 of this proof, we conclude that the point $\left(E_{x j}, E_{y j}\right)$ corresponding to the optimal tuple belongs both to the $x$ line and to the $y$-line. Thus, this point coincides with the intersection of these two lines.

In general, the $x$-line has the form $y-E_{y}=k_{x} \cdot\left(x-E_{x}\right)$. The $y$-line has the form $x-E_{x}=k_{y} \cdot\left(y-E_{y}\right)$, i.e., equivalently, $y-E_{y}=\frac{1}{k_{y}} \cdot\left(x-E_{x}\right)$. Both lines pass through the same point $\left(E_{x}, E_{y}\right)$, but their slopes are, in general, different: $k_{x}$ for the $x$-line and $\frac{1}{k_{y}}$ for the $y$-line. Thus, these lines coincide if and only if $k_{x}=\frac{1}{k_{y}}$, i.e., if and only if $k_{x} \cdot k_{y}=1$.

In general, $\rho \leq 1$. Here, $\rho=\frac{C}{\sqrt{V_{x}} \cdot \sqrt{V_{y}}}$; thus, $\rho=$ $\sqrt{k_{x} \cdot k_{y}}$, so $k_{x} \cdot k_{y} \leq 1$. If $k_{x} \cdot k_{y}<1$, then $k_{x} \cdot k_{y} \neq 1$ and thus, the $x$-line and the $y$-line are different. So, the intersection of these two lines is a single point $\left(E_{x}, E_{y}\right)$. If $k_{x} \cdot k_{y}=1$, this means that $\rho=1$, and all the points $\left(E_{x j}, E_{y j}\right)$ are on the same straight line - this is the case we have considered above.

\section{ACKNOWLEDGMENT}

The author is thankful to Vladik Kreinovich and to the anonymous referees for their guidance and suggestions.

\section{REFERENCES}

[1] A. Jalal-Kamali and V. Kreinovich, Estimating Correlation under Interval Uncertainty, University of Texas at El Paso, Department of Computer Science, Technical Report UTEP-CS-11-57, 2011, http://www.cs.utep.edu/vladik/tr11-57.pdf

[2] L. Longpré, G. Xiang, V. Kreinovich, and E. Freudenthal, "Interval Approach to Preserving Privacy in Statistical Databases: Related Challenges and Algorithms of Computational Statistics”, In: V. Gorodetsky, I. Kotenko, and V. A. Skormin (eds.), Proc. Int'l Conf. "Math. Methods, Models and Architectures for Computer Networks Security” MMMACNS-07, St. Petersburg, Russia, September 13-15, 2007, Springer Lecture Notes in Computer Science, 2007, Vol. CCIS-1, pp. 346-361.

[3] H. T. Nguyen, V. Kreinovich, B. Wu, and G. Xiang, Computing Statistics under Interval and Fuzzy Uncertainty, Springer Verlag, New York, 2012.

[4] H. T. Nguyen and E. A. Walker, A first course in fuzzy logic, CRC Press, Boca Raton, Florida, 2005.

[5] S. Rabinovich, Measurement Errors and Uncertainties: Theory and Practice, Springer Verlag, New York, 2005.

[6] G. Xiang and V. Kreinovich, "Estimating Variance under Interval and Fuzzy Uncertainty: Case of Hierarchical Estimation", In: P. Melin, O. Castillo, L. T. Aguilar, J. Kacprzyk, and W. Pedrycz (eds.), Foundations of Fuzzy Logic and Soft Computing, Proc. World Congress of the Int'1 Fuzzy Systems Association IFSA'2007, Cancun, Mexico, June 18-21, 2007, Springer Lecture Notes on Artificial Intelligence, 2007, Vol. 4529, pp. 3-12. 